\title{
Les combattants de la « mort certaine ». Les sens du sacrifice à l'horizon de la Grande Guerre
}

\section{François Lagrange}

\section{OpenEdition}

1 Journals

\section{Édition électronique}

URL : http://journals.openedition.org/conflits/2113

DOI : $10.4000 /$ conflits. 2113

ISSN : $1777-5345$

Éditeur :

CCLS - Centre d'études sur les conflits lilberté et sécurité, L'Harmattan

\section{Édition imprimée}

Date de publication : 1 novembre 2006

Pagination : 63-81

ISBN : 2-296-01436-4

ISSN : 1157-996X

\section{Référence électronique}

François Lagrange, «Les combattants de la « mort certaine ». Les sens du sacrifice à l'horizon de la Grande Guerre », Cultures \& Conflits [En ligne], 63 | automne 2006, mis en ligne le 05 décembre 2006 consulté le 30 mars 2021. URL : http://journals.openedition.org/conflits/2113 ; DOI : https://doi.org/ $10.4000 /$ conflits. 2113

Ce document a été généré automatiquement le 30 mars 2021.

Creative Commons License 


\title{
Les combattants de la « mort certaine ». Les sens du sacrifice à l'horizon de la Grande Guerre
}

\author{
François Lagrange
}

«En ces lieux l'on ne sait comment mourir se nomme,Tant ce fut une offrande à quoi chacun consent ${ }^{1}{ }^{\prime}$.

1 La notion de sacrifice a été employée par les autorités, les combattants et les populations civiles pour tenter de donner sens à l'épreuve de la Grande Guerre. L'historien y recourt pour définir certaines convictions essentielles des hommes du front :

« Nombre de croyants exaltent l'héroïsme du sacrifice, avec pour les catholiques, le désir d'imiter le Christ. De la même manière que celui-ci a souffert sa Passion pour le salut de l'humanité, le soldat chrétien meurt à la guerre pour sauver son pays ${ }^{2}$ ".

2 Il existe de nombreuses variantes, parfois complètement laïcisées, de ce motif. Le sacrifice des combattants de la Grande Guerre n'a apparemment rien en commun avec la mort volontaire des kamikazes (et moins encore avec les «hommes ou femmesbombes ${ }^{3}$ » de la seconde moitié du xxe siècle ou du xxie siècle commençant). La Grande Guerre semble même un cas limite, où, dans les liens ambigus entre sacrifice de soi et suicide, tout va au sacrifice et rien au suicide ${ }^{4}$. Nous voudrions, dans le cadre français de l'expérience de la Grande Guerre, dépasser cette impression initiale en examinant plus avant les éléments éventuels reliant sacrifice (de soi) et mort volontaire.

3 Nous procéderons en deux temps: d'abord une analyse des réflexions, antérieures à 1914, d'auteurs militaires notables sur le rôle du sacrifice à la guerre, ensuite l'examen des rares cas connus concrets de sacrifice de soi confinant à la mort volontaire au cours de la Grande Guerre. La difficulté de l'enquête - les sources sont disparates et dispersées - et les limites propres d'un article n'autorisent qu'un sommaire travail de défrichement.

Les théoriciens de la « mort certaine » avant 1914 
4 La pensée militaire française des années précédant la Grande Guerre a été l'objet de plusieurs études ${ }^{5}$ qui montrent la vivacité des débats et la pluralité des opinions. Deux auteurs se distinguent par leur vision radicale du sacrifice: le général Cardot et le lieutenant-colonel Montaigne.

Lucien Cardot ${ }^{6}$ est une figure importante avant 1914. Introducteur de Clausewitz à l'Ecole supérieure de guerre (ESG), il en propose une interprétation - discutable - qui a marqué ses auditeurs français ${ }^{7}$. Il martèle la définition clausewitzienne de la guerre, duel de deux volontés, et accorde une place prépondérante aux forces morales. Ayant suivi les manœuvres russes en 1882 et en 1895, Cardot devient l'admirateur de l'armée russe et de ses méthodes. Il se réfère fréquemment au général et théoricien militaire russe M.I. Dragomirov ${ }^{8}$. Le style de Cardot peut surprendre, mais celui-ci est pris au sérieux par ses collègues et bien noté par ses chefs ${ }^{9}$. Discutant les orientations de l'armée française, Cardot écrit un ouvrage polémique : Hérésies et apostasies militaires de notre temps ${ }^{10}$. La question du sacrifice et de son rôle décisif à la guerre y est posée.

Dans des propos à tonalité réactionnaire ${ }^{11}$, Cardot en vient à définir la vérité cardinale, selon lui, de la guerre : «Il faut trouver le moyen de conduire les gens à la mort, sinon, il n'y a plus de guerre possible ; ce moyen, je le connais ; il est dans l'esprit de sacrifice, et non ailleurs ${ }^{12}$ ». Il a recours à des tournures fort crues : «L'homme qui veut faire la guerre doit faire le sacrifice de sa peau, et tant que ce sacrifice n'est pas accompli sur sa personne, tant que sa propre peau est intacte, il ne peut pas s'en aller ${ }^{13}$ ! ». Il suit de près les idées de Dragomirov ${ }^{14}:$ " Nous ne devons pas oublier que notre mission est de tuer, en nous faisant tuer. [...] Il faut donc savoir tuer, tout en étant prêt à périr soimême ${ }^{15}$ ». Pour Cardot (et Dragomirov), faire la guerre, c'est aller à la mort. Le faire accepter aux hommes implique une logique du sacrifice, d'inspiration religieuse ${ }^{16}$. Si Cardot n'est pas isolé dans l'éloge du sacrifice, ses collègues sont ordinairement plus retenus dans la formulation. Ainsi, le capitaine Gilbert ${ }^{17}$ prône le sacrifice, mais au nom d'une collectivité presque abstraite, en l'occurrence, la nation, et ne se situe donc pas explicitement dans une perspective religieuse : «[...] enfin et surtout elle [la guerre] exige le sacrifice constant, total, absolu de l'individu à la collectivité ${ }^{18}$ ». Le général Langlois ${ }^{19}$ pense de même, mais enrobe davantage son expression : " De tous temps la guerre s'est payée cher et c'est avec le moral qu'on lutte, avec le moral qu'on gagne, en attaquant, pourvu que l'offensive prenne la forme qui convient à des conditions nouvelles ${ }^{20} »$.

7 Ces nuances dans les propos n'empêchent pas un accord fondamental : que le moteur soit la religion ou le patriotisme (ils ne sont pas exclusifs l'un de l'autre), la caractéristique d'une bonne troupe est son aptitude à aller au sacrifice. Les pertes prouvent un moral élevé, comme l'affirme Cardot:

«Les pertes ? - c'est bien, en effet, le prix dont on paye chaque pas en avant, car on n'avance qu'à coups d'hommes ; vaincre, c'est avancer et tout dépend du prix qu'on voudra y mettre. Ce sont les braves semés sur la route qui, en effet, ouvrent le chemin aux autres... ${ }^{21} »$.

8 Sur le plan tactique, l'évitement du choc avec l'ennemi est contraire à la nature de la guerre ${ }^{22}$, à « notre vieille religion de l'honneur et du sacrifice généreusement offert [...] ${ }^{23}$ !».

9 Cette vision de la guerre, avec son volet esthétique et incantatoire ${ }^{24}$, ne cesse de rappeler le rôle central du sacrifice. Cardot élabore un schéma dans lequel celui des 
deux adversaires qui a le plus de volonté, qui a le moins peur de la mort et qui le montre en prenant l'initiative (autrement dit qui attaque), gagne :

«Et quel est notre acte? La destruction - beaucoup plus exactement la démoralisation de l'adversaire - donc, et tout d'abord, produisons au grand jour cette force morale qui remplit nos cœurs; affirmons hautement à l'ennemi que notre mépris du danger et de la mort, que notre esprit de sacrifice l'emporte... ${ }^{25}$ ».

Le plus résolu, le plus disposé au sacrifice, et donc à la mort, l'emporte absolument par effet simple et radical d'une confrontation d'intensité des volontés : «[...] la guerre c'est le sacrifice ; la victoire appartient au plus brave, au plus résolu, à celui qui sait le mieux se faire tuer, à celui qui l'emporte dans la sublime surenchère du sacrifice ${ }^{26}$ !", paraphrase exaltée d'aphorismes de Dragomirov: «Ne crains pas de périr, si difficile que soit l'affaire ; tu es sûr de battre l'autre ${ }^{27} » ;$ « [...] On n'arrive au but que lorsqu'on est décidé à périr plutôt que d'en avoir le démenti ${ }^{28}$ ».

$11 \mathrm{Au}$ final, la représentation que Cardot se fait de la guerre comme phénomène moral et sacrificiel acquiert la force d'un dogme. La supériorité morale donne la victoire, l'obtention de la première et de la seconde implique une acceptation enthousiaste du sacrifice de soi. La mort recherchée est l'horizon du bon soldat et du bon officier.

Sans avoir l'importance de Cardot dans l'institution militaire, le lieutenant-colonel Montaigne ${ }^{29}$, qui en est intellectuellement proche ${ }^{30}$, rencontre un certain écho, ses publications étant assez nombreuses peu avant $1914{ }^{31}$. Lui aussi traite de l'usage militaire du sacrifice, par exemple durant la guerre russo-japonaise de 1904-1905 (négligée par Cardot).

13 Montaigne cite Dragomirov, sans étendre son admiration à l'armée russe. Il juge que ses revers proviennent d'un manque d'intensité dans le sacrifice, domaine où les Japonais ont excellé :

«"Le soldat japonais combat pour une cause sainte, l'avenir de la patrie; il a derrière lui la presse, la nation tout entière; les siens l'engagent à se sacrifier pour son pays". [...] Aussi, avant de rejoindre l'armée, le soldat japonais a fait le sacrifice de sa vie, et il dit aux siens, en les quittant, non au revoir, mais adieu. Dans son fanatisme patriotique, il ne rêve pas de retours triomphants et d'acclamations enthousiastes, il rêve de mourir pour la patrie ${ }^{32}$ ".

14 Cette complète acceptation par les soldats et les officiers nippons d'une mort quasi certaine stimule leur désir de victoire et fait jouer à leur profit la dynamique morale du sacrifice. Montaigne est fasciné par cet enthousiasme - jamais assimilé à un suicide qu'il illustre d'anecdotes :

«Paroles du colonel Acki avant l'assaut de Port-Arthur: "Notre brave colonne d'assaut ne doit pas seulement être prête à mourir; elle doit être certaine de mourir". Les hommes de la mort certaine, cette appellation plut au régiment ${ }^{33}$ ».

15 Les combattants ne sont pas seuls; ils sont portés dans leur élan vers la mort par la société entière dans la même ferveur patriotique. Ici s'amorce un décalage entre Cardot et Montaigne : le premier relève d'une sensibilité catholique traditionnelle. Le second, à partir du cas japonais, évolue vers une religion patriotique englobante, qui s'émancipe des références chrétiennes et possède une sorte d'avant-goût totalitaire ${ }^{34}$.

Montaigne voit dans le comportement nippon une source d'inspiration :

« [...] cette fureur d'immolation du soldat japonais est une apparition qui aurait dû frapper de stupeur nos cerveaux qui ne pensent qu'à la paix, de honte nos cours qui ne battent plus que pour le plaisir. [...] Le ferment actif du courage, c'est la passion. 
Les Japonais ont vaincu parce qu'ils étaient religieux et qu'ils aimaient la patrie

35 !».

L'expression "fureur d'immolation » connote sombrement le registre du sacrifice. Le ton se fait polémique, l'intention de Montaigne est claire : la logique japonaise de la «mort certaine » n'est pas pour lui qu'un simple phénomène exotique, il le propose au contraire comme modèle aux Français - même s'il reste assez flou sur ses modalités d'adaptation.

Le dernier élément à examiner concerne non pas un théoricien mais un futur praticien, et du plus haut niveau : le général de Castelnau ${ }^{36}$. Une récente biographie ${ }^{37}$ relate dans le détail ses propos oraux, connus sous le nom révélateur d'» homélie de la mort ${ }^{38} »$. Le 2 juillet 1914, à la IIe Armée, Castelnau critique un exercice militaire qui vient d'avoir lieu. Il interroge un officier supérieur sur ses dispositions :

« Tout à coup, l'interrompant, il [Castelnau] s'écria : "Mais enfin, colonel, où voulezvous mourir ?" Interloqué, le colonel esquissa un sourire et répondit : "Mais, mon général, je n'ai pas du tout l'intention de mourir" [Castelnau reprend] : "Il ne s'agit pas de cela. Je vous demande : où voulez-vous mourir? En d'autres termes : où avezvous assis votre ligne principale de résistance, celle que vous défendrez jusqu'à la mort ?" [...] "L'honneur militaire n'est pas l'honneur mondain. Il ne se satisfait pas au premier sang. Le devoir qu'il nous impose n'a qu'une limite, c'est la mort... [...] Dans toute action défensive, il y a une ligne qu'il n'est pas permis de dépasser... Derrière est le gouffre où sombre notre honneur. Cette limite d'extrême résistance, c'est l'endroit où on se fait tuer. On peut mourir n'importe où, on ne se fait tuer que là. Le choix de cette position est d'une importance capitale, car elle constitue la clé de chaque bataille". "[...] Il y a plus encore : il faut savoir comment mourir. La mort d'un soldat ne doit pas être un vain sacrifice, une sorte d'acte passif rappelant la résignation du gladiateur terrassé, s'offrant lui-même au couteau. Cette suprême ressource du combattant, je la vois comme l'explosion de toutes ses forces, le feu à la poudrière. Lorsqu'il n'y a plus qu'à mourir, il reste encore à mourir puissamment, et pour cela, il faut organiser solidement et judicieusement la position de résistance sur le terrain choisi [...]" ${ }^{39} "$.

Et Castelnau de conclure :

«"Le colonel Aimé avait raison de me répondre qu'il ne voulait pas mourir. Ce qu'il voulait, c'était vaincre. La mort ne sauve que l'honneur, la victoire sauve le pays. Mais la ferme décision de mourir plutôt que de reculer donne la mesure de notre force morale, de cette force qui fait seule la valeur d'une troupe. Cultivons-la, messieurs, dans notre cœur, dans le cœur de nos soldats, cette qualité qui engendre les héros et qui, à l'heure de la bataille, assurera la suprématie de nos armes" 40 ».

Comment ne pas être sensible à la comparaison de l'ultime effort du combattant avec une " explosion de toutes ses forces, le feu à la poudrière "? Il s'agit d'une image et il faut certes éviter l'anachronisme, ces mots n'en acquièrent pas moins, en ce début de xxie siècle, une résonance troublante.

Tels Cardot et Montaigne, Castelnau valorise le sacrifice de soi : vaincra le plus résolu à mettre son existence en jeu, sans espoir de survivre (parmi les officiers comme parmi les soldats). Castelnau introduit un autre paramètre; la « mort certaine " s'inscrit dans le contexte d'une action défensive ultime, différant profondément de la dynamique offensive de Cardot et de Montaigne. La détermination à se sacrifier n'est plus le seul moteur, la capacité à choisir le bon moment, celui qui donne le maximum d'efficacité à sa mort, est non moins important. Il y a combinaison d'une dimension presque religieuse, inconditionnée ${ }^{41}$, et d'une conjecture rationnelle sur l'utilité tactique. Le sacrifice ne doit pas être vain. 
22 La réaction du colonel interlocuteur de Castelnau prouve qu'une conception radicale du sacrifice est loin d'être acceptée par tous ${ }^{42}$. On peut se demander si son influence s'exerce au-delà de la sphère, capitale mais étroite, des spéculations de théoriciens et de grands chefs assez éloignés des réalités du terrain. Le milieu militaire dans lequel évolue Castelnau n'apparaît pas imprégné de l'enthousiasme auto-immolateur (sur le modèle nippon) cher à Montaigne. Castelnau lui-même atténue au final la rudesse de son message.

23 Ces aperçus de l'idéologie du sacrifice de soi chez certains responsables militaires français ${ }^{43}$ avant 1914 contiennent plusieurs enseignements :

24 - le thème n'est pas une création rétrospective. Il est lié à une vision de la guerre comme duel moral de deux volontés. La victoire va à l'armée préparée à l'effort le plus extrême, à celle qui compte le plus de combattants prêts à sacrifier leur vie. D'éminentes personnalités militaires adoptent cette conception et veulent la populariser. La «mort certaine» - acceptée, choisie, anticipée, plutôt que « volontaire » au sens strict - n'est jamais assimilée à un suicide, le registre lexical et sémantique est celui du sacrifice.

25 - il n'existe pas de discours unitaire du sacrifice de soi. Cardot l'adosse à une religiosité chrétienne typée. Montaigne sacrifie l'individu au pays dans le contexte d'une religion patriotique totalisante, potentiellement assez éloignée du christianisme. Cardot et Montaigne insistent sur l'intensité de la volonté de se sacrifier comme clef de la victoire. Castelnau intègre un calcul rationnel sur le rendement tactique défensif escompté du sacrifice de soi.

26 - aucun auteur, malgré les connotations et références religieuses, n'envisage la « mort certaine » sous l'angle des avantages spirituels personnels de celui qui va mourir. Les bénéficiaires du sacrifice sont en priorité les autres : les "camarades » ou "frères » soldats, l'armée, la patrie. Ce sacrifice altruiste n'est pas mis en relation avec le salut individuel.

27 Il est instructif de comparer ces orientations avec celles, contemporaines, de la jeune pensée sociologique. Dans Le Suicide, publié en $1897^{44}$, Durkheim adopte du phénomène une définition large qui fait sa place au militaire :

«Le soldat qui court au-devant d'une mort certaine pour sauver son régiment ne veut pas mourir, et pourtant n'est-il pas l'auteur de sa propre mort au même titre que l'industriel ou le commerçant qui se tuent pour échapper aux hontes de la faillite ${ }^{45}$ ?».

Aussi, parmi diverses catégories, Durkheim établit-il celle du « suicide altruiste ${ }^{46} »:$ « si l'homme se tue, ce n'est pas parce qu'il s'en arroge le droit, mais, ce qui est bien différent, parce qu'il en a le devoir ${ }^{47} »$. La référence militaire est explicite :

«La première qualité du soldat est une sorte d'impersonnalité que l'on ne rencontre nulle part, au même degré, dans la vie civile. Il faut qu'il soit exercé à faire peu de cas de sa personne, puisqu'il doit être prêt à en faire le sacrifice dès qu'il en a reçu l'ordre ${ }^{48}$ ».

Durkheim affine sa typologie en distinguant un "suicide altruiste obligatoire » - bien ajusté au contexte militaire - et d'autres formes au "caractère plus facultatif ${ }^{49}$ ». Le contraste entre Durkheim et les théoriciens militaires est extrême : pour le premier, le sacrifice de soi à la guerre est une variante du suicide ; pour les seconds, l'autosacrifice n'est jamais rapporté, ni de près ni de loin, au suicide. On mesure à cette aune combien l'observation et l'interprétation de cas réels s'avèrent délicates. 
Approches mémorielles éparses de morts problématiques témoignages sur des cas concrets et documentés de sacrifice de soi durant la Grande Guerre pose problème: point d'études systématiques sur le sujet. Témoins de Jean Norton $\mathrm{Cru}^{50}$, ouvrage déjà ancien, ne nourrit guère le dossier. Les carnets, journaux personnels, mémoires mais aussi les ouvrages de fiction à analyser constituent une masse énorme, exigeant une longue et méthodique investigation. Faute de temps et de place, force est de se limiter à une approche impressionniste et suggestive ${ }^{51}$.

Un récent travail universitaire sur Les Suicides durant la Première Guerre mondiale ${ }^{52}$, reprenant la typologie de Durkheim, note seulement trois cas de « suicides altruistes » :

« Le capitaine [...] protesta contre cette attaque organisée en dépit de tout bon sens et vouée à un échec certain, mais, mis en demeure d'obéir, il s'élança et tomba foudroyé après avoir fait quelques pas ${ }^{53}$ ».

«On nous a dit que le capitaine Ancelin, sûr d'être tué, s'était pourtant une seconde fois jeté contre les fils de fer intacts, parce qu'il en avait reçu l'ordre : on nous a dit aussi qu'il était un officier admirable, et mieux encore, un homme de cœur ${ }^{54}$ ".

«A la 17e compagnie, le lieutenant Sibille, voyant qu'on est obligé de battre en retraite, s'élance seul avec son revolver et des grenades et se fait tuer ${ }^{55}$ au milieu des Boches ${ }^{56}$ ".

Tirés de trois ouvrages différents, ces exemples de sacrifices de soi, situés au début du conflit, se ressemblent. Ils concernent le même type de population : des officiers allant à une mort quasi inévitable ${ }^{57}$, fin consentie, mais non, à strictement parler, volontaire. Par deux fois, est explicitement rappelé l'ordre supérieur qui motive leur décision ${ }^{58}$. Trois points peuvent donc être relevés, concernant les penseurs militaires d'avant 1914 :

- la sécheresse prosaïque du compte rendu de ces sacrifices diffère singulièrement de la rhétorique échauffée des théoriciens, le contraste est même spectaculaire ${ }^{59}$.

- l'influence du comportement de ces officiers sur leurs troupes demeure incertaine. L'effet d'entraînement moral paraît limité, voire nul. L'exemple du chef ne suscite pas de mimétisme sacrificiel collectif.

- - aucun récit n'évalue l'impact sur l'ennemi de l'action de ces officiers. Le profit tactique du sacrifice n'est pas envisagé.

La taille réduite de l'échantillon ${ }^{60}$ ne permet pas d'aller plus loin. La mémoire de certains morts célèbres de la Grande Guerre ouvre d'autres pistes.

C'est ainsi que Paul Léautaud et Daniel Halévy s'interrogent sur la mort exemplaire ${ }^{61} \mathrm{de}$ Péguy :

« Nous venons à parler de Péguy. Je lui dis carrément qu'il m'a toujours agacé, et que sa mort aussi m'agace, en bravache, s'offrant volontairement, etc. Mort aussi niaise qu'inutile. Daniel Halévy ${ }^{62}$ me dit qu'il y a tout lieu de croire qu'il y a eu dans cette mort quelque chose d'un suicide. Après cela, il n'a pas du tout l'air de mésestimer la mort sur un champ de bataille ${ }^{63}$ ».

Cet échange qui conduit à relire le témoignage connu de V. Boudon ${ }^{64}$ sur la fin de Péguy ${ }^{65}$, le 5 septembre 1914 dans l'après-midi à Villeroy. L'artillerie française a localement pris l'avantage et les fantassins français amorcent une contre-attaque, épisode du vaste mouvement menant à la victoire de la Marne :

«"Couchez-vous! hurle Péguy, et feu à volonté", mais lui reste debout, la lorgnette à la main, dirigeant notre tir, héroïque dans l'enfer. [...] Péguy est toujours debout, malgré nos cris de: "Couchez-vous !" glorieux fou dans sa bravoure. [...] Et il se dresse, comme un défi à la mitraille, semblant appeler cette mort qu'il glorifiait 
dans ses vers. Au même instant, une balle meurtrière brise ce noble front. Il est tombé, sur le côté, sans un cri, dans une plainte sourde, ayant eu l'ultime vision de la victoire tant espérée et enfin proche [...]. [L]es fumées des incendies qui rougeoient, alors que les canons hurlent à la mort, montent comme un encens de cette terre de sacrifice... ${ }^{66} "$.

Péguy veut maintenir ses hommes dans l'action malgré les pertes. A cette fin, il s'engage clairement sur la voie d'une mort quasi certaine (il n'est pas seul: Boudon décrit, au même moment, dans des termes presque identiques, la mort d'un autre officier, le lieutenant de Cornillère) : n'ordonne-t-il pas à ses hommes de se coucher pour éviter le feu ennemi alors qu'il refuse de le faire lui-même? Boudon, bien que très favorable à son chef, ne cache pas une certaine distance - admirative - envers ce comportement, tout en soulignant, y compris dans d'autres passages, le souci constant de Péguy de motiver ses subordonnés. La valeur exemplaire du sacrifice de soi paraît ici mieux attestée que dans les trois cas précédents. Le texte de Boudon se conclut d'ailleurs un peu plus loin par la citation du fameux poème de Péguy « Prière pour nous autres charnels ${ }^{67}$ », enraciné dans la thématique du sacrifice et de la guerre juste. Un autre effet se dégage à la lecture complète de l'ouvrage de Boudon : la mort de Péguy reçoit une sorte de sanctification stratégique car elle s'inscrit dans le cadre de la victoire de la Marne. Son sacrifice s'en trouve - plus ou moins consciemment rehaussé.

Une autre piste mémorielle, bien présente dans les milieux militaires ${ }^{68}$, mène aux saint-cyriens ayant juré d'aller au combat «en casoar et gants blancs ${ }^{69}$ ». La source imprimée la plus accessible est l'ouvrage En casoar et gants blancs ${ }^{70}$ de PaluelMarmont ${ }^{71}$, paru en 1928, préfacé par le général Gouraud ${ }^{72}$. Etabli à l'aide de divers témoignages et lettres ${ }^{73}$, ce recueil de faits d'armes de saint-cyriens vise ouvertement à l'édification de la jeunesse. Il comporte dix-huit récits : le premier rappelle le serment, les quinze suivants les actions héroïques, durant la Grande Guerre, de saint-cyriens des promotions 1912-1914 de Montmirail et 1913-1914 de La Croix du drapeau, les deux derniers évoquent des épisodes ultérieurs dans les colonies.

La première nouvelle, « Le Baptême au crépuscule ${ }^{74}$ ", décrit l'Ecole spéciale militaire à l'extrême fin du mois de juillet 1914. A cause de la tension internationale, les autorités de l'Ecole suspendent les traditions saint-cyriennes. Le «triomphe» (qui marque la fin du séjour à l'Ecole d'une promotion) de la Montmirail est annulé. Le "baptême de promotion" (à l'occasion duquel une promotion reçoit officiellement son nom, ses membres passant alors, suivant les usages, de l'appellation d'» hommes " à celle d'» officiers » ${ }^{75}$ ) de La Croix du drapeau se déroule dans la discrétion, à un horaire inhabituel, en soirée. A son issue survient le serment :

« - Oui, dit Allard-Méeus, jurons de monter la première fois à l'assaut en casoar et en gants blancs.Et tous ceux qui se trouvaient là prêtèrent ce serment ${ }^{76}$ ».

Retenons les deux éléments suivants :

- - ceux qui ont prêté serment ne sont pas très nombreux ${ }^{77}$, une vingtaine ${ }^{78}$, d'après des estimations récentes (la promotion de Montmirail compte 477 membres, La Croix du drapeau, $522^{79}$ ).

- l'ensemble du récit ne confère pas à cette décision un caractère aberrant (dû au culte du " panache ", etc.). Elle apparaît, du point de vue des (jeunes) saint-cyriens, comme une compensation à l'escamotage de leurs rites, à la suite de l'actualité exceptionnelle. La compression des cérémonies d'usage appelle un rééquilibrage : le port, lors du premier assaut, de signes traditionnels saint-cyriens (une fois sortis de l'ESM, les jeunes officiers 
portent l'uniforme réglementaire de l'arme dans laquelle ils servent ; on ne combat théoriquement pas en gants blancs, ni encore moins avec le casoar appartenant au « Grand Uniforme ${ }^{80}$ »de l'ESM).

Il est alors possible d'analyser les quinze chroniques de saint-cyriens dans la Grande Guerre. Six d'entre elles mettent en scène le sacrifice de soi avec présomption de "mort certaine ", voire volontaire ${ }^{81}$.

Le premier récit En casoar ${ }^{82}$ [1] porte sur Allard-Méeus, à l'origine du serment (présenté en admirateur de la légendaire charge de Reichshoffen ${ }^{83}$ ). Sa fin, le 22 août 1914, est brossée en deux temps. Allard-Méeus s'expose une première fois aux balles, de toute sa haute taille, pour amener un homme apeuré à reprendre le combat. La mort n'est pas loin :

«Alors, pour électriser les siens, il s'amusa, enleva son shako, et, le tenant à bout de bras :

- Les balles, dit-il, voici comment on les attrape.Pour lui donner raison, une balle troua sa coiffure. Il éclata de rire. Ses hommes, transportés, s'élancèrent.

En avant ! cria-t-il encore.

Mais son casoar vacilla au bout de sa main... Son bras croula. Allard-Méeus s'abattit $84 »$.

La prise de risque volontaire et répétée est manifeste. L'effet moral positif sur les troupes est indiqué. Le résultat tactique de l'engagement n'est pas abordé.

Le deuxième récit [2] fait écho au précédent, comme le suggère son titre : « ... Et gants blancs ${ }^{85}$ ». Il raconte la mort d'Alain de Fayolle (il a prêté le serment), le 22 août également, alors que ses troupes sont prises sous le feu allemand:

«Ceux qui étaient le plus près de lui fixèrent leur regard dans le sien, comme pour implorer de ce magnifique officier de vingt ans, qui était leur chef, l'énergie nécessaire. Lui, comprit ce qu'ils demandaient.

Voici, dit-il.

De sa musette, Alain de Fayolle tira son casoar qu'il piqua sur son képi, et ses gants blancs, qu'il enfila sans hâte et boutonna. Puis, se dressant soudain, grandi de toute la hauteur de son plumet:

En avant, mes enfants !... Pour la France !

Et, presque aussitôt, il tomba ${ }^{86}$ ".

4 L'aspect volontaire de la démarche de Fayolle est indubitable, le risque de mort, évident. Le sacrifice de soi doit galvaniser les hommes et ces derniers sont montrés en attente d'un geste de leur supérieur. Pas plus que pour Allard-Méeus, un gain tactique concret n'est mentionné.

Ces deux récits sont archétypaux. Les quatre autres, dans l'ordre, sont moins démonstratifs. Trois Allemands ${ }^{87}$ [3] raconte la mort de Le Brizec à la fin de la bataille de la Marne. Submergé par le nombre après avoir tué trois ennemis, il continue à lutter, tombe et meurt des suites de ses blessures le lendemain, 12 septembre. Dans Trois Balles ${ }^{88}[4]$ est exposé le cas de Cotelle : prenant de très grands risques, celui-ci est blessé à trois reprises le 22 août. Ne pouvant plus se déplacer, il reste sur place, refusant le secours d'un sous-officier: on le retrouve mort le lendemain. Je ne crains rien ${ }^{89}$ [5] relate la mort du sous-lieutenant de Blottefière, tué le 22 août par des tireurs allemands dissimulés alors qu'il s'expose. La Passerelle ${ }^{90}[6]$ se conclut par la mort de Loriot de la Salle, aux confins de la France et de la Belgique, le 14 novembre 1914 : tenant à sortir en tête d'une tranchée, il est immédiatement fauché. 
Ces textes sont plus elliptiques, plus ambigus, l'influence du sacrifice de soi sur les troupes est moins nette que dans les deux premières relations [1] et [2]. Il faudrait développer, en affinant la part des faits et celles des représentations mais la place nous manque. Un point paraît acquis: le serment n'est pas le moteur du sacrifice (qui s'explique par les conditions du combat), il relève plutôt du décor (chacun, sauf [6], porte tout ou partie de sa tenue de l'ESM).

Après l'étude de ces dix morts, il est possible d'esquisser une typologie provisoire du sacrifice de soi pendant la Grande Guerre selon trois critères :

- l'acceptation d'une mort certaine (ou très probable), consentie, voire recherchée ;

- la volonté de se servir du sacrifice de soi pour renforcer le moral des troupes (hiérarchie oblige) ;

- le rendement tactique ou stratégique du sacrifice.

La mort de Péguy constitue une combinaison optimale : il va au devant du danger létal, son action incite ses hommes à poursuivre le combat, les opérations auxquelles il prend part sont un maillon utile dans la chaîne d'engagements aboutissant à la victoire de la Marne. Les sacrifices saint-cyriens sont souvent inspirés (sauf [4]) par le souci de motiver les troupes, leur caractère délibéré est patent, mais leur portée tactique n'est pas claire et quatre d'entre eux ont pour arrière-plan la défaite de Charleroi. Les trois cas de Barthas, de Genevoix et de Laby, plus tardifs, présentent le dosage le plus faible : la «mort certaine " est à deux reprises le produit d'un ordre venu d'en haut, jugé discutable. L'utilité militaire de ces morts semble nulle. L'impact sur les hommes, difficile à évaluer, paraît neutre ou déprimant.

Ces différences sensibles ne reflèteraient-elles pas une évolution de la guerre peu favorable aux formes radicales d'autosacrifice sur le front occidental? Déjà pendant la phase de mouvement (morts de Péguy et des saint-cyriens), les armes modernes (les mitrailleuses, etc.) tuent massivement à distance, réduisant drastiquement l'impact sur l'ennemi du sacrifice de soi. Le phénomène s'accentue fin 1914, avec la guerre de siège, usante, de petits groupes, d'escouades ${ }^{91}$ à mobilité réduite, ou, à une autre échelle, guerre d'artilleurs à plus longue distance, qui ne se voient pas, ou mal: si même il y avait volonté d'autosacrifice, où trouver un point d'application pertinent? La destruction de l'ennemi dépasse souvent les possibilités d'un individu, serait-il prêt à se sacrifier. Le grand nombre des victimes dévalue d'une certaine façon la mort, volontaire ou non, héroïque ou non. Il est aisé de se faire tuer, difficile de se faire tuer utilement. Il faut tenir, ce qui relève d'une autre vision de la guerre, dont Gérard Fassy constate l'émergence chez les saint-cyriens: « ... pour vaincre, il faut vivre ${ }^{92}$ ». Les morts saint-cyriennes du début de la guerre sont alors passibles de critiques : Gouraud concède que le serment " a coûté cher ${ }^{93}$ " tout en insistant sur sa valeur exemplaire.

A cette approche - fragmentaire, ne portant que sur un très mince échantillon - de cas concrets de sacrifice de soi dans la Grande Guerre, s'ajoute un dernier élément, que l'on doit à Maurice Halbwachs, disciple de Durkheim. Publiant en 1930 Les Causes du suicide ${ }^{94}$, il conteste l'assimilation qui est faite entre sacrifice et suicide ${ }^{95}:$ « Nous approchons ici d'un ordre de faits qui ressemble au suicide. Pourtant l'opinion l'en distingue [...]. Le soldat tombé sur le champ de bataille, et qui a été au-devant d'une mort certaine, n'est pas un suicidé ${ }^{9} \%$.

Comment ne pas supposer Halbwachs influencé par les réactions sur le sujet pendant et après le conflit ${ }^{97}$ ? Son insistance sur le caractère visible et socialisé du sacrifice, par 
opposition au suicide ${ }^{98}$, n'éclaire-t-elle pas en partie le serment des saint-cyriens, sorte de rite minimal d'entrée dans le sacrifice ${ }^{99}$ ? A l'inverse, observe Halbwachs,

« Même lorsqu'un soldat se fait tuer volontairement pour son pays, [...] pour que cet acte produise ce qu'on en attend il faut que la société se l'approprie, qu'elle le consacre par une commémoration qui s'entourera nécessairement de formes conventionnelles... ${ }^{100} \%$.

Cette absence de cérémonial n'est-elle pas nette dans les trois cas mentionnés par Barthas, Genevoix (malgré un effort du commandement) et Laby?

Au terme de cette enquête, il paraît utile, avec les précautions d'usage, de proposer quelques hypothèses situant ce type singulier de mort par rapport au vaste registre, englobant et complexe, du sacrifice.

Des auteurs abordent la question avant 1914. Il semble y avoir eu, au début du conflit, un potentiel de "mort certaine ", les rares éléments concrets glanés incitent à le penser. Mais les conditions matérielles et organisationnelles du combat, dès la brève guerre de mouvement et plus encore après, dans les tranchées, ne se prêtent guère à sa réalisation. Ce modèle d'un sacrifice de soi de forte intensité paraît lié à une élite militaire professionnelle préparant une guerre courte. La longueur du conflit, les modalités de la guerre de siège, l'immensité des effectifs et des pertes, l'ont rapidement marginalisé ${ }^{101}$.

Nombre d'historiens de la Grande Guerre ${ }^{102}$ ont, par ailleurs, constaté la force durable du consentement au conflit des populations impliquées, notamment en France. La thématique du sacrifice de soi en est une forme privilégiée d'expression. Les archives du contrôle postal ${ }^{103}$ le montrent abondamment. Ce discours du sacrifice, par accord progressif, souvent implicite ${ }^{104}$, entre combattants et dirigeants (militaires ${ }^{105}$ et politiques ${ }^{106}$ ), acquiert un profil nettement différent de celui de la «mort certaine » : il ne s'agit plus de mourir à coup sûr, mais d'accepter durablement le risque de mort (et de blessure, et de souffrance, et de séparation d'avec les siens, etc. Le sacrifice redéfini possède donc un centre, la mort, mais aussi une large périphérie). Les combattants français, en temps de "mort de masse ${ }^{107}$ ", estiment ce niveau de sacrifice de soi suffisant, la victoire finale (et les bienfaits espérés pour leurs proches et le pays) demeurant la garante de la validité ${ }^{108}$ des sacrifices acceptés ${ }^{109}$.

Il resterait à s'interroger sur la façon dont les sociétés européennes, notamment occidentales, ont réagi en profondeur, après la guerre, à l'ampleur des sacrifices endurés, toutes formes confondues. Sur le plan français, observateurs et historiens soulignent ce que le pacifisme viscéral de la population et des dirigeants, durant l'entre-deux-guerres, doit au refus de consentir de nouveau à des sacrifices comparables à ceux de la Grande Guerre ${ }^{110}$. Sur le plan plus strictement militaire, apparaissent des conceptions très éloignées des doctrinaires d'avant 1914, destinées à se développer jusqu'à nos jours, et que résument bien les propos du célèbre général américain Patton :

« [...] lui qui expliquait en substance à ses troupes que le devoir du soldat n'est pas de mourir pour sa patrie, mais de faire en sorte que d'autres soldats, dans l'autre camp, meurent pour la leur : vérité crue et cynique, aux antipodes du serment de 1914 , mais dont nul ne saurait contester le bon sens ${ }^{111}{ }^{\prime}$. 


\section{NOTES}

1.Comtesse de Noailles, «Verdun », Prévost E., Dornier C. (dir.), Le Livre épique, anthologie des poèmes de la Grande Guerre, Paris, librairie Chapelot, 1920, p. 133.

2.Boniface X., « Croyances et convictions », Lagrange F. (dir.), Inventaire de la Grande Guerre, Paris, Universalis, 2005, p. 318.

3.Stroumsa G.G., La Fin du sacrifice, les mutations religieuses de l'Antiquité tardive, Paris, Odile Jacob, 2005, p. 135.

4.Sur la différenciation entre sacrifice et suicide, voir Pinguet M., La Mort volontaire au Japon, Paris, Gallimard, 1984, p. 115. S'opposent ainsi, dans une logique chrétienne, la mort du Christ (sacrifice) et celle de Caton d'Utique (suicide).

5.Commandant Goya M., La Chair et l'Acier, l'invention de la guerre moderne (1914-1918), Paris, Taillandier, 2004 ; Queloz D., « La pensée militaire française et les enseignements de la guerre des Boers ", Stratégique n 84 , 2001, paru en 2004, pp. 61-84 ; Lagrange F., "Le culte de l'offensive : logique et paradoxes des penseurs militaires d'avant 1914 ", Cahier d'études et de recherches du musée de l'Armée (CERMA) n5, 1904-1914: de la guerre pensée à la guerre sur le terrain, à paraître en 2006.

6.Lucien Cardot (1838-1920). Saint-cyrien, il participe à la guerre de 1870-1871, il est blessé le 18 août 1870. Dossier SHAT 10 YD 586. Breveté en 1881, conférences à l'ESG de 1884 à 1886 (puis de nouveau en 1900), général de brigade en 1895 .

7.Raymond Aron, Penser la guerre, Clausewitz, t. II, L'âge planétaire, Gallimard, 1976, pp. 28, 34-35 et pp. 315-317 ainsi que le lieutenant-colonel Montaigne, Etudes sur la guerre, Paris/Nancy, librairie Berger-Levrault, 1911, p. 3.

8.M.I. Dragomirov (1830-1905) a été directeur de l'Ecole de guerre russe de 1878 à 1889. Ses écrits traduits en français ont exercé une influence certaine, voir de Lastours $S$., «Les grands traits de la pensée militaire russe », Stratégique n49, 1991, pp. 79-80. La transcription de son nom la plus fréquente, en France, dans les années précédant la Grande Guerre, est " Dragomiroff », mais on trouve parfois « Dragomirof ».

9.Voir son dossier d'inspection, par exemple en 1900, dossier SHAT 10 YD 586.

10.Général Cardot, Hérésies et apostasies militaires de notre temps, Paris/Nancy, BergerLevrault et Cie, 1908. Cardot a soixante-dix ans, sa carrière militaire est finie, il peut s'exprimer librement.

11.Joseph de Maistre est souvent cité, voir Cardot, op. cit., par exemple p. 230, p. 310 et p. 438.

12.Ibid., p. 89.

13.Ibid., pp. 453-454.

14.Qu'il qualifie de "mon cher maître, Dragomirov », ibid., p. VI.

15.Général Dragomiroff, Manuel de préparation des troupes au combat, Préparation de la compagnie, Librairie militaire de L. Baudoin et Cie, 1885, p. 12 (traduction française de la cinquième édition du manuel de Dragomirov parue en Russie la même année).

16.Cardot, op. cit., p. XVIII.

17.Le capitaine Gilbert, polytechnicien ayant quitté l'armée pour raisons de santé, est devenu journaliste spécialisé dans les questions militaires, proche de l'Etat-major. Dimitry Queloz, op. cit., p.64, note 9.

18.Capitaine G. Gilbert, La Guerre sud-africaine, Berger-Levrault, 1902, préface du général Bonnal, p. 502. 
19.Le Général Langlois a été professeur à l'ESG, membre du Conseil supérieur de la guerre (CSG) et bientôt, à sa retraite, sénateur. D. Queloz, op. cit., p. 64, note 8 .

20.Général Langlois, Enseignements de deux guerres récentes..., Lavauzelle, s.d., p. 148. Le terme en romain est en italique dans le texte.

21. Cardot, op. cit., p. 452.

22.Ibid., p. XXXVI.

23.Ibid., p. LXI.

24.Alain a critiqué cette dimension esthétique de la guerre, voir Alain, « Du beau », Mars ou la guerre jugée, 1921, rééd. coll. « Folio/essais », Paris, Gallimard, 1995, pp. 46-48. 25. Cardot, op. cit., p. 104.

26.Ibid. p. 457.

27.Dragomiroff, op. cit., p. 136.

28.Ibid., p. 137.

29.Peu d'informations sur Jean-Baptiste Montaigne : saint-cyrien, breveté, né en 1857. Voir Cru J.N., Témoins, essai d'analyse et de critique des souvenirs de combattants édités en français de 1915 à 1928, Paris, Les Etincelles, 1929 ; réédition Nancy, Presses universitaires de Nancy, 1993, p. 77. Auteur de plusieurs ouvrages sur les problèmes militaires avant 1914, il est cité dans les travaux scientifiques traitant de ces questions, par exemple, outre Cru J.N., op. cit., voir Contamine H., op. cit., p. 172 ; Doise J., Vaïsse M., Diplomatie et outil militaire 1871-1991, Paris, Imprimerie nationale, 1987, rééed. Points/Histoire, 1992, p. 685 , note $\mathrm{n}^{\circ} 101$, Goya M., op. cit., notamment p. 48 et pp. 130-132.

30.Voir son appréciation, très élogieuse, des conférences de Cardot à l'ESG : lieutenantcolonel Montaigne, Etudes sur la guerre, Berger-Levrault, 1911, p. 3 : « Et la parole du commandant Cardot assura ma foi ».

31. Notamment Montaigne, Etudes sur la guerre, op. cit. et Vaincre, Esquisse d'une doctrine de la guerre basée sur la connaissance de l'Homme et sur la Morale, Berger-Levrault, 3 volumes, 1913.

32.Montaigne, Vaincre, op. cit., pp. 125-126.

33.Ibid., p. 126, suite de la note 1 p. 125.

34.Admiration similaire pour les Japonais chez Ludendorff, le véritable chef de guerre allemand d'août 1916 à octobre 1918, puis allié politique de Hitler dans les années 1920. Voir Ludendorff, La Guerre totale, Paris, Flammarion, 1937, p. 22.

35.Montaigne, Vaincre, op. cit., pp. 127-128.

36.1851-1944. Saint-cyrien, breveté, désigné sous-chef d'état major de l'armée en 1911 pour assister Joffre nouvellement nommé, membre du Conseil supérieur de la guerre (CSG), commande plusieurs armées françaises tout au long de la Grande Guerre, sauf en 1915-1916 où il seconde de nouveau Joffre. Ses convictions religieuses catholiques lui valent le surnom de « capucin botté ». Député du « Bloc national » de 1919 à 1924.

37.Général Yves Gras, Castelnau ou l'art de commander, Paris, Denoël, 1990.

38.La formule est d'un témoin, René Brice, voir Gras Y., ibid., p. 143.

39.Phrase célèbre qu'on trouve, légèrement modifiée, dans Nobécourt J., Une histoire politique de l'armée, 1, 1919-1942, de Pétain à Pétain, Paris, Le Seuil, 1967, p. 155.

40.Gras Y., op. cit., pp. 142-143.

41. Castelnau reste très discret, en public, sur cet arrière-plan religieux : peut-on y voir un souci de ne pas heurter les autorités politiques laïques de la IIIe République?

42.Un témoin de « l'homélie de la mort » en convient explicitement, voir Gras Y., op. cit., p. 143. 
43.Il faudrait comparer à l'échelle européenne. On sait déjà par Dragomirov qu'il y a ressemblance avec les Russes, dans un environnement politique et religieux autre. 44.Durkheim E., Le Suicide, 1897, 12e éd., coll. « Quadrige », Paris, PUF, 2004.

45.Ibid., p. 4.

46.Ibid., pp. 233-263. Les deux autres types sont le « suicide égoïste » et le « suicide anomique $»$.

47.Ibid., p. 236.

48.Ibid., p. 254.

49.Ibid., pp. 238 et 239. Durkheim parle également de « suicide héroïque », p. 262.

50.Cru J.N., Témoins, op. cit. Cru critique tout « bourrage de crâne » et se méfie des légendes sur l'héroïsme et le « panache ». Voir la table des matières p. 723. Son œuvre, qui a fait longtemps autorité, est actuellement controversée. Voir la mise au point de Smith L.V., « Jean Norton Cru et la subjectivité de l'objectivité », Becker J.J. (dir.), Histoire culturelle de la Grande Guerre, Paris, Armand Colin, 2005, pp. 89-100.

51.Voir Audoin-Rouzeau S., " Micro-histoire et histoire culturelle de la Grande Guerre : apports et limites d'une approche » Becker J.J. (dir.), op. cit., pp. 231-238.

52.Davion F., Les Suicides durant la Première Guerre mondiale, mémoire de maîtrise sous la direction de Stéphane Audoin-Rouzeau, université de Picardie Jules-Verne, 2004.

Rolland D., « Le suicide aux armées en 1914-1918 : une première approche quantitative globale », Cazals R., Picard E., Rolland D. (dir.), La Grande Guerre, Pratiques et expériences, Paris, Privat, 2005, pp. 269-279. Cet article n'aborde pas le « suicide altruiste ».

53.Barthas L., Les Carnets de guerre de Louis Barthas, tonnelier 1914-1918, introduction et postface de Rémy Cazals, Paris, La Découverte, 2003, p. 78 [vers le 17 décembre 1914].

54.Genevoix M., Ceux de 14, La boue, 25 décembre [1914]-5 janvier [1915], 1re éd., Paris, Flammarion, 1921, rééd. 1950, Paris, Points/Seuil, 1984, p. 479.

55.Expression ambiguë.

56.Les Carnets de l'aspirant Laby, Médecin dans les tranchées 28 juillet 1914-14 juillet 1919, avant-propos de S. Audoin-Rouzeau, texte préparé et annoté par S. Delaporte, Paris, Bayard, 2001, Paris, Hachette Littératures/Pluriel, 2003, p. 136.

57.Quasi inévitable seulement : le hasard aurait pu ne les laisser que (très) gravement blessés, voir Halbwachs M., Les Causes du suicide, 1930, rééd. Paris, PUF, 2005, p. 360 : " la mort d'un soldat qui se fait tuer volontairement pour son pays, d'un croyant qui meurt pour sa foi, dont il est d'ailleurs difficile de distinguer les mêmes actes accomplis par des hommes qui s'exposent seulement à un danger de mort, et qui meurent en effet, pour les mêmes causes ». 58.Voir Durkheim E., op. cit., p. 238, sur le « suicide altruiste obligatoire ». 59.Or si Barthas peut être considéré comme plutôt contestataire, ce n'est le cas ni de Genevoix ni de Laby.

60.Davion F., op. cit., pp. 106-107, reproduit un document iconographique étonnant sans le dater ni indiquer de numéro d'inventaire - du fonds de l'Historial de Péronne : une affiche en couleur rédigée en anglais faisant la publicité d'un film documentaire sur la guerre, The Kaiser's Challenge. Il s'agit du dessin d'un avion se jetant contre un zeppelin, l'hélice faisant exploser le dirigeable allemand dont l'équipage tombe pour partie dans le vide. L'annonce, portée sur l'affiche, l'explicite : « a french aviator forfeits his life in destroying a zeppelin killing 25 German soldiers. » (« un aviateur français sacrifie sa vie en détruisant un zeppelin, tuant 25 soldats allemands ", notre traduction). On songe naturellement aux kamikazes. La notice consacrée à ce film sur le site du New York Times indique que ce document est un montage, diffusé en 1914 aux Etats-Unis peu après le début de la guerre. La bataille aérienne est fabriquée de toute pièce. S'inspire- 
t-elle d'un fait de guerre réel ? Cette mise en scène indique que des formes radicales du sacrifice de soi peuvent affleurer dans le contexte de la guerre. Je remercie la conservation de l'Historial de Péronne qui, consultée le 21 mars 2006, m'a aimablement aiguillé vers le site Internet du New York Times : http://movies2.nytimes.com/gst/ movies/movie.html?v_id=236550.

61.Lanson G., Histoire illustrée de la littérature française, Paris, Hachette, 1923, t. 2, p. 410 : "La beauté foncière de ce tempérament tourmenté se dégagea au dernier jour, en septembre 1914, quand le lieutenant Péguy tomba héroïquement dans la bataille de la Marne ».

62.Proche de Péguy, notamment en 1901-1905, Laurent S., Daniel Halévy, Paris, Grasset, 2001, pp. 157-164.

63.Léautaud P., Journal littéraire, t. 1, « novembre 1893-juin 1928 », Paris, Le Mercure de France, 1986, pp. 1111-1112.

64.Boudon V., Avec Charles Péguy, De la Lorraine à la Marne, août-septembre 1914, préface de Barrès, Paris, Hachette, 1916. Une deuxième édition de l'ouvrage, parue en 1964, est intitulée Mon lieutenant Charles Péguy, juillet-septembre 1914, Paris, Albin Michel. Dans cet article nous nous référons à la version la plus ancienne. Boudon, simple soldat, est blessé le 6 septembre 1914.

65.Jean Norton Cru (op. cit., pp. 96-97) critique Boudon pour son « ignorance naïve » du combat et de la guerre. Il lui reproche aussi (surtout ?) la préface de Barrès. Il ne met pas en doute la véracité des faits sur Péguy.

66.Boudon V., op. cit., pp. 144-147.

67. "Couchés dessus le sol à la face de Dieu », tiré du poème "Prière pour nous autres charnels ", 1913, qui ouvre le livre de Boudon.

68.La promotion de 1963-1965 de l'Ecole spéciale militaire a pris le nom du « Serment de 1914 ", cinquante ans après, voir Collectif, Les Saint-cyriens, vocation et destinées, musée de l'Armée, 2002, p. 230.

69.Le rapport complexe entre réalité et représentation incite mutatis mutandis à la comparaison avec les « jeunes de Langemarck » allemands, à l'automne 1914, voir Mosse G.L., De la Grande Guerre au totalitarisme, la brutalisation des sociétés européennes, 1990, Paris, Hachettes/Littératures, 2001, pp. 83-87.

70.Paluel-Marmont, En casoar et gants blancs, préface du général Gouraud, La nouvelle société d'édition, 1928. D'autres auteurs ont déjà mentionné les faits, Cru J.N., op. cit., pp. 460 et 477 durant ou juste après la guerre.

71.Albert P. Paluel-Marmont, 104e promotion 1919-1920, Des croix de guerre, « homme de lettres ", est mentionné dans l'Annuaire des anciens élèves de l'Ecole spéciale militaire de 1953. Je remercie le commandant Souprayen et Madame Plichard, du Musée du Souvenir des écoles de Saint-Cyr Coëtquidan, pour ces renseignements. PaluelMarmont est donc proche des jeunes saint-cyriens de 1914 et des faits qu'il relate, comme le rappelle Gouraud (p. 9). Il a également écrit dans un autre registre à en croire les titres de ses ouvrages déjà publiés en 1928 : Fille du sud et Le Visage perdu.

72.Ancien saint-cyrien, collaborateur de Lyautey, Gouraud (1867-1946) a commandé aux Dardanelles en 1915 - il y a reçu de graves blessures aux jambes et au bras qui l'ont laissé infirme, amputé du bras droit - puis la IVe Armée en Champagne de décembre 1915 à décembre 1916 et de juin 1917 à la fin de la guerre. Haut commissaire de la France en Syrie de 1919 à 1923, gouverneur militaire de Paris de 1923 à 1937, Les Saintcyriens, vocation et destinées, op. cit., p. 138. Son statut de grand blessé mutilé en fait une figure vivante du sacrifice, d'où son choix comme préfacier. 
73.La valeur documentaire stricte du livre est difficile à évaluer. Le texte est repris pour partie par Paluel-Marmont A. dans Nos grandes écoles : Saint-Cyr, La nouvelle sociétés d'édition, 1930, cité comme source par colonel Jacques Vernet, général Pierre Gourmen, général Jean Boÿ, colonel Pierre Jacob, Yves Gourmen, dans Saint-Cyr, l'Ecole spéciale militaire, Lavauzelle, 2002. Nous intéresse surtout ici la façon dont est représenté le sacrifice de soi.

74.Paluel-Marmont A., op. cit., pp. 13-30.

75.Boÿ J., « La naissance des traditions de l'ESM et leur évolution jusqu'à nos jours ", Cahier d'études et de recherches du musée de l'Armée (CERMA) n4, Saint-Cyr, la société militaire, la société française, 2002, pp. 115-135.

76.Paluel-Marmont A., op. cit., p. 30. 77 . Une légende, dénoncée par le colonel Michel Camus, Histoire des saint-Cyriens, Lavauzelle, 1980, p. 185, prête le serment à toute une promotion.

77.Une légende, dénoncée par le colonel Michel Camus, Histoire des saint-Cyriens, Lavauzelle, 1980, p. 185, prête le serment à toute une promotion.

78.Les Saint-cyriens, vocation et destinées, op. cit., p. 27 et p. 130, précisions apportées par le commandant Christophe Gué.

79.Fassy G., « Les jeunes saint-cyriens dans la Grande Guerre », CERMA nº 4, op. cit., p. 182.

80.Général Jean Boÿ, op. cit., CERMA n4, op. cit., pp. 119-120.

81.Sept autres cas se concluent par une mort soit sans équivoque soit non décrite.

82.Paluel-Marmont A., op. cit., pp. 31-42. Pour faciliter les comparaisons, nous numérotons ces nouvelles de [1] à [6].

83.Paluel-Marmont A., op. cit., pp. 34-35. Voir Roth F., La Guerre de 1870, Paris, Fayard, 1990, p. 48 : « la tragique et inutile chevauchée de la brigade Michel ».

84.Ibid., p. 42.

85.Ibid., pp. 43-52.

86.Ibid., p. 52. Voir colonel Jacques Vernet, général Pierre Gourmen, général Jean Boÿ et al., op. cit., p. 543.

87.Ibid., pp. 53-61.

88.Ibid., pp. 75-86.

89.Ibid., pp. 113-122.

90.Ibid., pp. 191-200.

91.Mosse G.L., op. cit., p. 9.

92.Fassy G., op. cit., CERMA $\mathrm{n}^{\circ} 4$, op. cit., p. 179. Il relève quelques faits héroïques dans les Balkans en 1917 pp. 179-180, mais rien de comparable au début de la guerre.

93.Paluel-Marmont A., op. cit., préface du général Gouraud, p. 7. Des contestations ultérieures ont été plus brutales. Général Marc Défourneaux, Guerre des armes, guerre des hommes, ADDIM, 1994, pp. 35-36 : «Ces sacrifices héroïques ont-ils eu pour effet d'exalter le patriotisme des survivants et, par là, leur ardeur au combat ? Ou bien, au contraire, ont-ils bêtement privé la patrie de soldats de valeur au moment où elle en avait le plus besoin?».

94.Livre réédité aux PUF en 2005.

95.Halbwachs M., Les Causes du suicide, op. cit., pp. 339-360, « La définition du suicide. Suicide et sacrifice ».

96.Ibid., p. 340. Halbwachs conteste pareillement, contre Durkheim, que le martyr chrétien soit un suicidé. 
97.Becker A., « Maurice Halbwachs et l'Alsace, de la Grande Guerre à l'université de Strasbourg ", Audoin-Rouzeau S., Becker A. et al. (dir.), La Politique et la guerre, pour comprendre le xxe siècle européen. Hommage à Jean-Jacques Becker, Paris, éditions Agnès Viénot/Noésis, 2002, pp. 284-300, notamment pp. 294-299.

98.Ibid., pp. 356-357.

99.Voir l'entrée dans le sacrifice de Mauss M., Hubert H., Mélanges d'histoire des religions, librairie Félix Alcan, 2e éd., 1929, « Essai sur la nature et la fonction du sacrifice », pp. 22-23, « Le schème du sacrifice ", reprise d'un article paru en 1899, op. cit., p. 1.

100.Ibid., p. 357.

101.Mosse G.L., op. cit., pp. 145-178.

102.Becker J.J., 1914, Comment les Français sont entrés dans la guerre, Paris, Presses de la Fondation nationale des sciences politiques, 1977. L'Année 1914, Armand Colin, 2004, pp. 253-262. Voir aussi Audoin-Rouzeau S., Becker A., 14-18, retrouver la guerre, Paris, Gallimard, 2000, pp.111-120. Le sujet reste débattu, parfois âprement : Rousseau F., La Guerre censurée : une histoire des combattants européens de 14-18, Paris, Le Seuil, 1999 ; Prost A., Winter J., Penser la Grande Guerre, Un essai d'historiographie, coll. « Points/Histoire », Paris, Le Seuil, 2004, pp. 140-143 ; Cochet F., Survivre au front 1914-1918, Les Poilus entre contrainte et consentement, 14-18 éditions, 2005.

103.Qui débute en 1915 ; Cochet A., L'Opinion et le moral des soldats en 1916, d'après les archives du Contrôle postal, Nanterre, université de Paris-X Nanterre, 1985 ; Nicot J., Les Poilus ont la parole, lettres du front :1917-1918, Bruxelles, éditions Complexe, 1998, pp. 7-13 et pp. 263-279 ; Cabanes B., « Ce que dit le contrôle postal », Prochasson C., Rasmussen A. (dir.), Vrai et faux dans la Grande Guerre, Paris, La Découverte, 2004, pp. 55-75.

104.Mauss M., Hubert H., op. cit., p. 127 : « Au fond, il n'y a peut-être pas de sacrifice qui n'ait quelque chose de contractuel ». Voir Smith L.V., Between Mutiny and Obedience, The Case of the French Fifth Infantry Division during World War I, Princetown, Princeton University Press, 1994, pp. 16-17 et p. 64. Il établit ce qu'en français, on pourrait appeler un " principe de proportionnalité » des sacrifices et des résultats.

105.Pédroncini G., Les Mutineries de 1917, Paris, PUF, 2e éd. mise à jour, 1983, pp.

311-312.

106.Déclaration ministérielle du 20 décembre 1917, Clemenceau G., Discours de guerre, Paris, PUF, 1968, p. 131.

107. Mosse G. L., op. cit., p. 9.

108. Hubert H., et Mauss M., op. cit., p. 127 : « Le sacrifice se présente donc sous un double aspect. C'est un acte utile et c'est une obligation. Le désintéressement s'y mêle à l'intérêt ». Quand il y a doute sur le commandement et la victoire, il y a remise en cause de la pertinence du sacrifice, d'où les mutineries ; Pétain, La Crise morale et militaire de 1917, Nouvelles Editions latines, 1966, p. 46, en est très conscient (document rédigée par Pétain dès 1925, ibid., p. 14).

109.En Allemagne, des refus de sacrifice de soi interviennent dans la défaite. Au niveau des chefs, avec Guillaume II, Baechler C., Guillaume II d'Allemagne, Paris, Fayard, 2003, p. 435. Au niveau des troupes, avec les marins allemands début novembre 1918, Vidil C., Les Mutineries de la Marine allemande 1917-1918, Paris, Payot, 1931, pp. 147-161 et Jardin P., Aux racines du mal,1918. Le déni de la défaite, Paris, Taillandier, 2005, pp. 311-410.

110.Becker J.J., Les Français dans la Grande Guerre, Paris, Robert Laffont, 1980, p. 305 ; Aron R., Le Spectateur engagé, entretiens avec Jean-Louis Missika et Dominique Wolton, Paris, Julliard, 1981, p. 58. Voir pour le domaine anglo-saxon et la Seconde Guerre mondiale, 
Fussell P., A la guerre, psychologie et comportements pendant la Seconde Guerre mondiale, Paris, Le Seuil, 1992, pp. 179-198. Voir également, notamment pour le domaine allemand, Mosse G.L., op. cit., pp. 229-231.

111. Général Marc Défourneaux, Guerre des armes, guerre des hommes, ADDIM, 1994, p. 36. Le général Défourneaux est polytechnicien. Peut-être Falkenhayn, en 1916 à Verdun, at-il amorcé un projet de ce genre, mais ce point est discuté, voir Krumeich G., « Verdun, un lieu pour une mémoire commune », Morizet J., Müller H. (dir.), Allemagne France, lieux et mémoire d'une histoire commune, Paris, Albin Michel, 1995, pp. 133-139 ; Afflerbach H., Falkenhayn, Politisches Denken und Handeln im Kaiserreich, Oldenburg, 1996, pp. 360-375 et pp. 543-545 ; Foley R.T., German Strategy and the Path to Verdun, Erich von Falkenhayn and the Development of Attrition, 1870-1916, Cambridge, Cambridge University Press, 2005, pp. 181-208.

\section{RÉSUMÉS}

Témoignages et analyses historiques assimilent souvent les épreuves des combattants de la Grande Guerre à des sacrifices. Rien ne les relie, à première vue, aux formes répertoriées de la mort volontaire (kamikazes ou attentats-suicides). Pourtant il existe des éléments qui permettent d'envisager, à la marge, par exemple dans le cadre français de l'expérience du premier conflit mondial, une certaine proximité entre discours du sacrifice de soi et mort volontaire. Une analyse des conceptions d'auteurs militaires notables avant 1914 restitue leur vision radicale du sacrifice de soi à la guerre, qui aboutit à valoriser la «mort certaine ». Une enquête complémentaire fait apparaître, au début de la Grande Guerre, quelques cas de " mort certaine », cas-limites peu nombreux mais significatifs qu'il importe de ne pas négliger dans une réflexion comparatiste sur le sens et les usages du sacrifice de soi.

Testimonies and historical analyses often identify the experiences of the combatants of the "Great War" to sacrifices. Nothing links them, at first looks, to the defined features of voluntary death (kamikazes or suicide-bombings). However some elements allow, at the margins, for example in the frame of the French experiences of WWI, to see common traits between the discourses on self-sacrifice on the one hand, voluntary death on the other. An analysis of the conceptions of military authors before 1914 allows reconstructing their radical vision of selfsacrifice in war leading to a valorisation of "certain death". A complementary investigation reveals, at the beginning of the "Great War", some cases of "certain death". Although limited in number, these borderline-cases are significant. They shall not be overlooked in the comparative approach of the different senses and practices of self-sacrifice.

\section{INDEX}

Thèmes : armée de terre (fr)

Index chronologique : 1914-1918

Index géographique : Allemagne, France

Mots-clés : conflits internationaux, guerre, kamikaze (missions suicide), Militaires 


\section{AUTEUR}

\section{FRANÇOIS LAGRANGE}

François Lagrange est responsable de la division de la recherche historique et de l'action pédagogique du musée de l'Armée et rédacteur en chef des Cahiers d'études et de recherches du musée de l'Armée. Il a dirigé l'Inventaire de la Grande Guerre, paru aux éditions Universalis en 2005. 